\title{
Software Transactional Memory for Dynamic-Sized Data Structures
}

\author{
Maurice Herlihy \\ Department of Computer Science \\ Brown University \\ Providence, RI 02912, USA \\ mph@cs.brown.edu
}

\author{
Mark Moir \\ Sun Microsystems Laboratories \\ 1 Network Drive \\ Burlington, MA 01803, USA \\ mark.moir@sun.com
}

\author{
Victor Luchangco \\ Sun Microsystems Laboratories \\ 1 Network Drive \\ Burlington, MA 01803, USA \\ victor.luchangco@sun.com \\ William N. Scherer III \\ Department of Computer Science \\ University of Rochester \\ Rochester, NY 14620, USA \\ scherer@cs.rochester.edu
}

\begin{abstract}
We propose a new form of software transactional memory (STM) designed to support dynamic-sized data structures, and we describe a novel non-blocking implementation. The non-blocking property we consider is obstruction-freedom. Obstruction-freedom is weaker than lock-freedom; as a result, it admits substantially simpler and more efficient implementations. A novel feature of our obstruction-free STM implementation is its use of modular contention managers to ensure progress in practice. We illustrate the utility of our dynamic STM with a straightforward implementation of an obstruction-free red-black tree, thereby demonstrating a sophisticated non-blocking dynamic data structure that would be difficult to implement by other means. We also present the results of simple preliminary performance experiments that demonstrate that an "early release" feature of our STM is useful for reducing contention, and that our STM lends itself to the effective use of modular contention managers.
\end{abstract}

\section{INTRODUCTION}

Using locks in programs for shared-memory multiprocessors introduces well-known software engineering problems. Coarse-grained locks, which protect relatively large amounts of data, generally do not scale: threads block one another even when they do not really interfere, and the lock becomes a source of contention. Fine-grained locks can mitigate these scalability problems, but they introduce software engineering problems as the locking conventions for guaranteeing correctness and avoiding deadlock become complex and error-prone. Locks also cause vulnerability to thread

Copyright is held by Sun Microsystems, Inc.

$P O D C^{\prime} 03$, July 13-16, 2003, Boston, Massachusetts, USA.

ACM 1-58113-708-7/03/0007. failures and delays. For example, a thread preempted while holding a lock will obstruct other threads.

Dynamic Software Transactional Memory (DSTM) is a low-level application programming interface (API) for synchronizing shared data without using locks. A transaction is a sequence of steps executed by a single thread. Transactions are atomic: each transaction either commits (it takes effect) or aborts (its effects are discarded). Transactions are linearizable [9]: they appear to take effect in a one-ata-time order. Transactional memory supports a computational model in which each thread announces the start of a transaction, executes a sequence of operations on shared objects, and then tries to commit the transaction. If the commit succeeds, the transaction's operations take effect; otherwise, they are discarded. Although transactional memory was originally proposed as a hardware architecture [8], there have been several proposals for non-blocking ${ }^{1}$ software transactional memory (STM) and similar constructs $[3,4$, $10,13,14,15]$.

We present the first dynamic STM. Prior STM designs required both the memory usage and the transactions to be defined statically in advance. In contrast, our new DSTM allows transactions and transactional objects to be created dynamically, and transactions may determine the sequence of objects to access based on the values observed in objects accessed earlier in the same transaction. As a result, DSTM is well suited to the implementation of dynamic-sized data structures such as lists and trees.

We have developed prototype implementations of DSTM in the $\mathrm{C}++$ and Java ${ }^{\mathrm{TM}}$ programming languages. In this paper, we focus on the Java version, which is considerably simpler because there is no need for explicit memory management. Our Java implementation uses an experimental prototype of Doug Lea's java .util . concurrent package [1] to call native compare-and-swap (CAS) operations.

\footnotetext{
${ }^{1}$ We use "non-blocking" broadly to include all progress conditions requiring that the failure or indefinite delay of a thread cannot prevent other threads from making progress, rather than as a synonym for "lock-free", as some authors prefer.
} 
Much of the simplicity of our implementation is due to our choice of non-blocking progress condition. A synchronization mechanism is obstruction-free [7] if any thread that runs by itself for long enough makes progress (which implies that a thread makes progress if it runs for long enough without encountering a synchronization conflict from a concurrent thread). Like stronger non-blocking progress conditions such as lock-freedom and wait-freedom, obstruction-freedom ensures that a halted thread cannot prevent other threads from making progress.

Unlike lock-freedom, obstruction-freedom does not rule out livelock; interfering concurrent threads may repeatedly prevent one another from making progress. Livelock is, of course, unacceptable. Nonetheless, we believe that there is great benefit in treating the mechanisms that ensure progress as a matter of policy, evaluated by their empirical effectiveness for a given application and execution environment. As demonstrated here and elsewhere $[7,11]$, compared to lockfreedom, obstruction-freedom admits substantially simpler implementations that are more efficient in the absence of synchronization conflicts among concurrent threads.

Obstruction-freedom also allows simple schemes for prioritizing transactions because it allows any transaction to abort any other transaction at any time. In particular, a high-priority transaction may always abort a low-priority transaction. In a lock-based approach, the high-priority transaction would be blocked if the low-priority transaction held a lock that the high-priority transaction required, resulting in priority inversion and intricate schemes to circumvent this inversion. On the other hand, in a lock-free implementation, the high-priority transaction may have to help the low-priority transaction complete in order to ensure that some transaction will complete.

Our obstruction-free DSTM implementation provides a simple open-ended mechanism for guaranteeing progress and prioritizing transactions. Specifically, one transaction can detect that it is about abort another before it does so. In this case, it consults a contention manager to determine whether it should abort the other transaction immediately or wait for some time to allow the other transaction a chance to complete. Contention managers in our implementation are modular: various contention management schemes can be implemented and "plugged in" without affecting the correctness of the transaction code. Thus we can design, implement and verify an obstruction-free data structure once, and then vary the contention managers to provide the desired progress guarantees and transaction prioritization. These contention managers can exploit information about time, operating systems services, scheduling, hardware environments, and other details about the system and execution environment, as well as programmer-supplied information. These practical sources of information have been largely neglected in the literature on lock-free synchronization. We believe that this approach will yield simpler and more efficient concurrent data structures, which will help accelerate their widespread acceptance and deployment.

Section 2 illustrates the use of DSTM through a series of simple examples. To evaluate the utility of DSTM for implementing complex data structures, we have also used it to implement an obstruction-free red-black tree. As far as we are aware, this red-black tree is the most complex nonblocking data structure achieved to date. Although our implementation is a reasonably straightforward transformation of a sequential implementation [6], it would be very difficult to construct such a non-blocking implementation from first principles. Indeed, it would be difficult to implement even a lock-based red-black tree that allows operations accessing different parts of the tree to proceed in parallel.

Section 3 describes how our STM detects synchronization conflicts and how transactions commit and abort, with an emphasis on how the obstruction-free property simplifies the underlying algorithm. In Section 4, we describe how our implementation interfaces with contention managers, which are responsible for ensuring progress. Section 5 describes some simple experiments conducted with our prototype DSTM implementation. Concluding remarks appear in Section 6. Code for our DSTM implementation, contention managers, and related experiments is publicly available [2].

\section{OVERVIEW AND EXAMPLES}

In this section, we illustrate the use of DSTM through a series of simple examples. DSTM manages a collection of transactional objects, which are accessed by transactions. A transaction is a short-lived, single-threaded computation that either commits or aborts. A transactional object is a container for a regular Java object. A transaction can access the contained object by opening the transactional object, and then reading or modifying the regular object. Changes to objects opened by a transaction are not seen outside the transaction until the transaction commits. If the transaction commits, then these changes take effect; otherwise, they are discarded.

Transactional objects can be created dynamically at any time. The creation and initialization of a transactional object is not performed as part of any transaction.

Concretely, the basic unit of parallel computation is the TMThread class, which extends regular Java threads. Like a regular Java thread, it provides a run() method that does all the work. In addition, the TMThread class provides additional methods for starting, committing or aborting transactions, and for checking on the status of a transaction. Threads can be created and destroyed dynamically.

Transactional objects are implemented by the TMObject class. To implement an atomic counter, one would create a new instance of a Counter class (not shown), and then create a TMObject to hold it:

$$
\begin{aligned}
& \text { Counter counter }=\text { new Counter }(0) ; \\
& \text { TMObject tmObject }=\text { new TMObject }(\text { counter }) ;
\end{aligned}
$$

Any class whose objects may be encapsulated within a transactional object must implement the TMCloneable interface. This interface requires the object to export a public clone () method that returns a new, logically disjoint copy of the object. DSTM uses this method when opening transactional objects, as described below. (DSTM guarantees that the object being cloned does not change during the cloning, so no synchronization is necessary in the clone() method.)

A thread calls beginTransaction() to start a transaction. Once it is started, a transaction is active until it is either committed or aborted.

While it is active, a transaction can access the encapsulated counter by calling open():

Counter counter = (Counter) tmobject. open (WRITE); counter.inc();// increment the counter 
The argument to open() is a constant indicating that the caller may modify the object. The open () method returns a copy of the encapsulated regular Java object ${ }^{2}$ created using that object's clone() method; we call this copy the transaction's version.

The thread can manipulate its version of an object by calling its methods in the usual way. DSTM guarantees that no other thread can access this version, so there is no need for further synchronization.

Note that a transaction's version is meaningful only during the lifetime of the transaction. References to versions should not be stored in other objects; only references to transactional objects are meaningful across transactions.

A thread attempts to commit its transaction by invoking commitTransaction(), which returns true if and only if the commit is successful. A thread may also abort its transaction by invoking abortTransaction().

We guarantee that successfully committed transactions are linearizable: they appear to execute in a one-at-a-time order. But what kind of consistency guarantee should we make for a transaction that eventually aborts? One might argue that it does not matter, as the transaction's changes to transactional objects are discarded anyway. However, synchronization conflicts could cause a transaction to observe inconsistencies among the objects it opens before it aborts. For example, while a transaction $T$ is executing, another transaction might modify objects that $T$ has already accessed as well as objects that $T$ will subsequently access. In this case, $T$ will see only partial effects of that transaction. Because transactions should appear to execute in isolation, observing such inconsistencies may cause a transaction to have unexpected side-effects, such as dereferencing a null pointer, array bounds violations, and so on.

DSTM addresses this problem by validating a transaction whenever it opens a transactional object. Validation consists of checking for synchronization conflicts, that is, whether any object opened by the transaction has since been opened in a conflicting mode by another transaction. If a synchronization conflict has occurred, open() throws a Denied exception instead of returning a value, indicating to the transaction that it cannot successfully commit in the future. The set of transactional objects opened before the first such exception is guaranteed to be consistent: open() returns the actual states of the objects at some recent instant. (Throwing an exception also allows the thread to avoid wasting effort by continuing the transaction.)

Ultimately, we would like DSTM to support nested transactions, so that a class whose methods use transactions can invoke from within a transaction methods of other classes that also use transactions. However, we have not acquired sufficient experience programming with DSTM to decide on the appropriate nesting semantics, so we do not specify this behavior for now.

\subsection{Extended Example}

Consider a linked list whose values are stored in increasing order. We will use this list to implement an integer set (class

\footnotetext{
${ }^{2}$ The open() method actually returns an object of class java. lang. Object, which we must explicitly cast back to class Counter.

${ }^{3}$ Our implementation does support a rudimentary form of nested transactions, but we do not use it in any of the examples discussed in this paper.
}



Figure 1: Integer Set Example

IntSet) that provides insert (), delete(), and member () methods. Relevant code excerpts are shown in Figure 1.

The IntSet class uses two types of objects: nodes and list elements; nodes are transactional objects (class TMDbject) that contain list elements (class List), which are regular Java objects. The List class has the following fields: value is the integer value, and next is the TMObject containing the next list element. We emphasize that next is a TMObject, not a list element, because this field must be meaningful across transactions. Because list elements are encapsulated within transactional objects, the List class implements the 
TMCloneable interface, providing a public clone() method.

The IntSet constructor allocates two sentinel nodes, containing list elements holding the minimum and maximum integer values (which we assume are never inserted or deleted). For brevity, we focus on insert(). This method takes an integer value; it returns true if the insertion takes place, and false if the value was already in the set. It first creates a new list element to hold the integer argument, and a new node to hold that list element. It then repeatedly retries the following transaction until it succeeds. The transaction traverses the list, maintaining a "current" node and a "previous" node. At the end of the traversal, the current node contains the smallest value in the list that is greater than or equal to the value being inserted. Depending on the value of the current node, the transaction either detects a duplicate or inserts the new node between the previous and current nodes, and then tries to commit. If the commit succeeds, the method returns; otherwise, it resumes the loop to retry the transaction.

An attractive feature of DSTM is that we can reason about this code almost as if it were sequential. The principal differences are the need to catch Denied exceptions and to retry transactions that fail to commit, and the need to distinguish between transactional nodes and non-transactional list elements. Note that after catching a Denied exception, we must still call commitTransaction() to terminate the transaction, even though it is guaranteed to fail.

\subsection{Conflict Reduction}

A transaction $A$ will typically fail to commit if a concurrent transaction $B$ opens an object already opened by $A$. Ultimately, it is the responsibility of the contention manager (discussed in Section 4) to ensure that conflicting transactions eventually do not overlap. Even so, the IntSet implementation just described introduces a number of unnecessary conflicts. For example, consider a transaction that calls member () to test whether a particular value is in the set, running concurrently with a transaction that calls insert () to insert a larger value. One transaction will cause the other to abort, since they will conflict on opening the first node of the list. Such a conflict is unnecessary, however, because the transaction inserting the value does not modify any of the nodes traversed by the other transaction. Designing the operations to avoid such conflicts reduces the need for contention management, and thereby generally improves performance and scalability.

DSTM provides several mechanisms for eliminating unneeded conflicts. One conventional mechanism is to allow transactions to open nodes in read-only mode, indicating that the transaction will not modify the object.

List list $=$ (List) node.open (READ);

Concurrent transactions that open the same transactional object for reading do not conflict. Because it is often difficult, especially in the face of aliasing, for a transaction to keep track of the objects it has opened, and in what mode each was opened, we allow a transaction to open an object several times, and in different modes.

The revised insert() (not shown) method walks down the list in read-only mode until it identifies which nodes to modify. It then "upgrades" its access from read-only to regular access by reopening that transactional object in WRITE mode. Read-only access is particularly useful for navigating

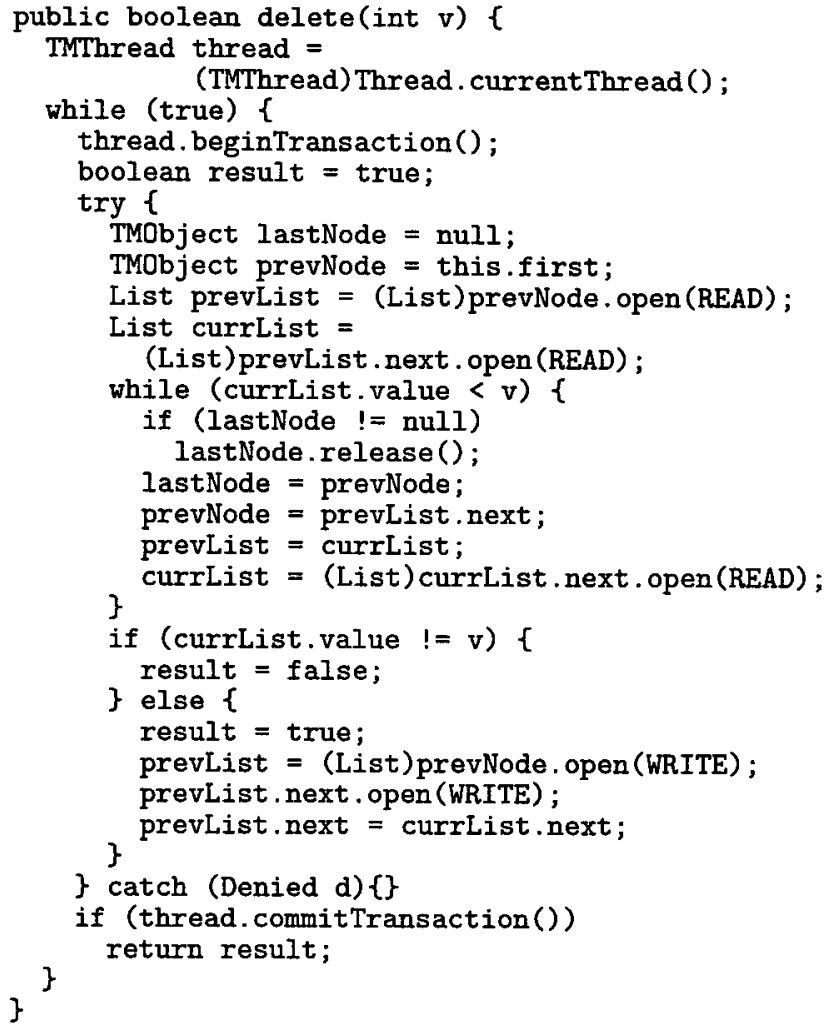

Figure 2: Delete method with early release

through tree-like data structures where all transactions pass through a common root, but most do not modify the root.

DSTM also provides a novel and more powerful (and more dangerous!) way to reduce conflicts. Before it commits, a transaction may release an object that it has opened in READ mode by invoking the release() method. Once an object has been released, other transactions accessing that object do not conflict with the releasing transaction over the released object. The programmer must ensure that subsequent changes by other transactions to released objects will not violate the linearizability of the releasing transaction. The danger here is similar to the problem mentioned earlier to motivate validation; releasing objects from a transaction causes future validations of that transaction to ignore the released objects. Therefore, as before, a transaction can observe inconsistent state. The effects in this case are potentially even worse because that transaction can actually commit, even though it is not linearizable.

In our IntSet example, releasing nodes is useful for navigating through the list with a minimum of conflicts, as shown in Figure 2. As a transaction traverses the list, opening each node in READ mode, it releases every node before its prev node. ${ }^{4}$ A transaction that adds an element to the list "upgrades" its access to the node to be modified by reopening that node in WRITE mode. A transaction that removes an element from the list opens in WRITE mode both the node to be modified and the node to be removed. It is easy to check that these steps preserve linearizability.

Because a transaction may open the same object several times, the DSTM matches, for each object, invocations of

\footnotetext{
${ }^{4}$ This is analogous to the technique of lock coupling (see [5], e.g.), but of course does not use any locks.
} 


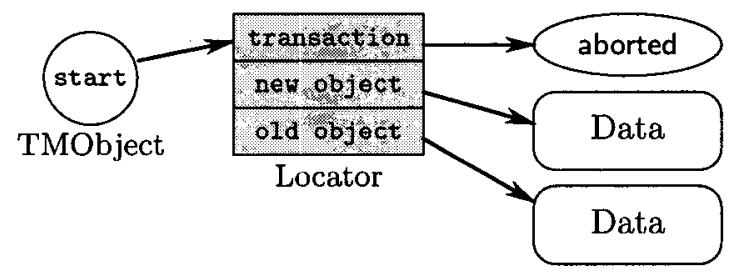

Figure 3: Transactional object structure

release () with invocations of open(READ); an object is not actually released until release() has been invoked as many times as open(READ) for that object. Objects opened in WRITE mode by a transaction cannot be released before the transaction commits; if a transaction opens an object in READ mode and then "upgrades" to WRITE mode, subsequent requests to release the object are silently ignored.

Clearly, the release facility must be used with care; careless use may violate transaction linearizability. Nevertheless, we have found it useful for designing shared pointer-based data structures such as lists and trees, in which a transaction reads its way through a complex structure.

\section{IMPLEMENTATION}

We now describe our DSTM implementation. A transaction object (class Transaction) has a status field that is initialized to be ACTIVE, and is later set to either COMMITTED or ABORTED using a CAS instruction. (CAS functionality is provided by the AtomicRef class in the experimental prototype of Doug Lea's java.util. concurrent package [1].)

\subsection{Opening a Transactional Object}

Recall that a transactional object (class TMObject) is a container for a regular Java object, which we call a version. Logically, each transactional object has three fields:

- transaction points to the transaction that most recently opened the transactional object in WRITE mode;

- oldobject points to an old object version; and

- newObject points to a new object version.

The current (i.e., most recently committed) version of a transactional object is determined by the status of the transaction that most recently opened the object in WRITE mode. If that transaction is committed, then the new object is the current version and the old object is meaningless. If the transaction is aborted, then the old object is the current version and the new object is meaningless. If the transaction is active, then the old object is the current version, and the new object is the active transaction's tentative version. This version will become current if the transaction commits successfully; otherwise, it will be discarded. Observe that, if several transactional objects have most recently been opened in WRITE mode by the same active transaction, then changing the status field of that transaction from ACTIVE

${ }^{5} \mathrm{~A}$ CAS $(a, e, n)$ instruction takes three parameters: an address $a$, an expected value $e$, and a new value $n$. If the value currently stored at address a matches the expected value $\theta$, then CAS stores the new value $\mathrm{n}$ at address a and returns true; we say that the CAS succeeds in this case. Otherwise, CAS returns false and does not modify the memory; we say that the CAS fails in this case.

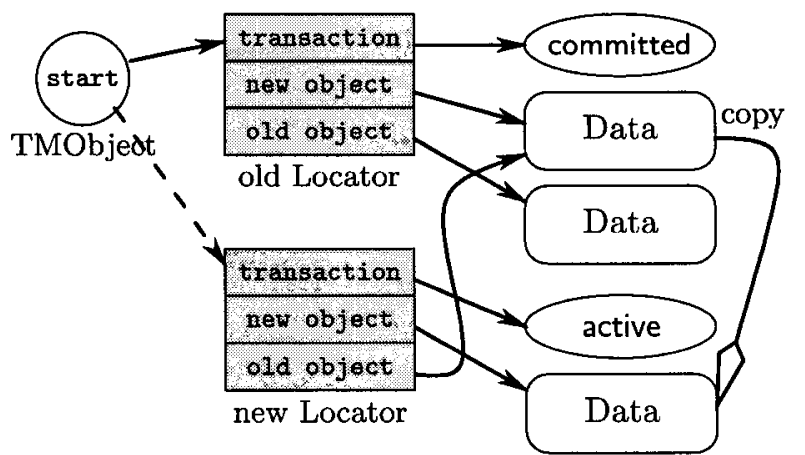

Figure 4: Opening transactional object after recent commit

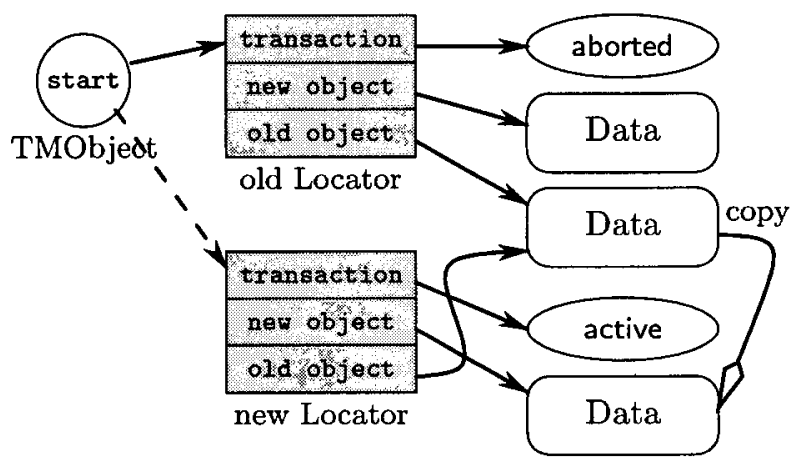

Figure 5: Opening transactional object after recent abort

to COMMITTED atomically changes the current version of each respective object from its old version to its new version; ${ }^{6}$ this is the essence of how atomic transactions are achieved in our implementation.

The interesting part of our implementation is how a transaction can safely open a transactional object without changing its current version (which should occur only when the transaction successfully commits). To achieve this, we need to atomically access the three fields mentioned above. However, current architectures do not generally provide hardware support for such atomic updates. Therefore, we introduce a level of indirection, whereby each TMObject has a single reference field start that points to a Locator object (Figure 3). The Locator object contains the three fields mentioned above: transaction points to the transaction that created the Locator, and oldObject and newObject point to the old and new object versions. This indirection allows us to change the three fields atomically by calling CAS to swing the start pointer from one Locator object to another.

We now explain in more detail how transaction $A$ opens a TMObject in WRITE mode. Let $B$ be the transaction that most recently opened the object in WRITE mode. $A$ prepares a new Locator object with transaction set to $A$. Suppose $B$ is committed. $A$ sets the new locator's oldobject field

\footnotetext{
${ }^{6}$ Because objects opened for reading by a transaction that successfully commits can change after the transaction successfully validates them but before it executes the CAS that changes its status, the transaction is linearized to the invocation of the commit, not to the point that the CAS succeeds. This point is subtle and we defer a complete discussion and the proof of linearizability to the full version of this paper.
} 
to the current newObject, and the new newObject field to a copy of the the current newObject (Figure 4). (Recall that every class that can be encapsulated by a transactional object must export a public clone() method.) $A$ then calls CAS to change the object's start field from $B$ 's old locator to $A$ 's new locator. If the CAS succeeds, the open() method returns the new version, which is now the transaction's tentative version of this object. $A$ can update that version without further synchronization. If the CAS fails, the transaction rereads the object's start field and retries. Suppose, instead, that $B$ is aborted. $A$ follows the same procedure, except that it sets the new locator's oldobject field to the current oldobject (Figure 5).

Finally, suppose $B$ is still active. Because $B$ may commit or abort before $A$ changes the object's start field, $A$ cannot determine which version is current at the moment its CAS succeeds. Thus, $A$ cannot safely choose a version to store in the oldobject field of its Locator. The beauty of obstruction-freedom is that $A$ does not need to guarantee progress to $B$, and can therefore resolve this dilemma by attempting to abort $B$ (by using CAS to change $B$ 's status field from ACTIVE to ABORTED) and ensuring that $B$ 's status field is either ABORTED or COMMI'TTED before proceeding (the change may have been effected by the action of some other transaction). This resolution also highlights an important property of our algorithm with respect to the integration of contention managers: Because $A$ can determine in advance that it will interfere with $B$, it can decide, based on the policy implemented by its contention manager (discussed in the next section), whether to abort $B$ or to give $B$ a chance to finish.

Read-only access is implemented in a slightly different way. When $A$ opens a transactional object $o$ for reading, it identifies the last committed version $v$ (possibly by aborting an active transaction) exactly as for write access. However, instead of installing a new Locator object, $A$ adds the pair $(o, v)$ to a thread-local read-only table.

To match invocations of open(READ) and release(), the transaction also maintains a counter for each pair in its readonly table. If an object is opened in READ mode when it already has an entry in the table, the transaction increments the corresponding counter instead of inserting a new pair. This counter is decremented by the release() method, and the pair is removed when the counter is reduced to zero.

\subsection{Validating and Committing a Transaction}

After open() has determined which version of an object to return, and before it actually returns that version, the DSTM must validate the calling transaction in order to ensure that the user transaction code can never observe an inconsistent state. Validation requires two steps:

1. For each pair $(o, v)$ in the calling thread's read-only

\footnotetext{
${ }^{7}$ Readers familiar with the use of CAS may be concerned about the ABA problem [12], in which a CAS operation fails to notice that the location it accesses has changed to a new value and then back to the original value, causing the CAS to succeed when it should have failed. This problem does not arise in our Java implementation, because garbage collection (GC) ensures that a Locator object does not get recycled until no thread has a pointer to it. While GC eliminates the $\mathrm{ABA}$ problem in this case, we caution the reader against assuming that the $A B A$ problem can never occur in environments that support GC.
}

table, verify that $v$ is still the most recently committed version of 0 .

\section{Check that the status field of the Transaction object remains ACTIVE.}

Committing a transaction requires two steps: validating the entries in the read-only table as described above, and calling CAS to attempt to change the status field of the Transaction object from ACTIVE to COMMITTED. 8

\subsection{Costs}

In the absence of synchronization conflicts, a transaction that opens $W$ objects for writing requires $W+1$ CAS operations: one for each open() call, and one to commit the transaction. Synchronization conflicts may require more CAS operations to abort other transactions. These are the only strong synchronization operations needed by our DSTM implementation: once open() returns an object version, there is no need for further synchronization to access that version. A transaction also incurs the cost of cloning objects opened for writing; cloning is achieved using simple load and store instructions because the DSTM ensures objects being cloned do not change during the cloning.

Validating a transaction that has opened $W$ objects for writing and $R$ objects for reading (that have not been released) requires $O(R)$ work. Because validation must be performed whenever an object is opened and when the transaction commits, the total overhead due to the DSTM implementation for a transaction that opens $R$ for reading and $W$ objects for writing is $O((R+W) R)$ plus the cost of copying each of the $W$ objects opened for writing once. Note that, in addition to reducing the potential for conflict, releasing objects opened for reading also reduces the overhead due to validation: released objects do not need to be validated. Thus, if at most $K$ objects are open for reading at any time, then the total overhead for a transaction is only $O((R+W) K)$ plus the cost of cloning the $W$ objects.

\section{CONTENTION MANAGEMENT}

Despite our advocacy of obstruction-free synchronization, we do not expect progress to take care of itself. On the contrary, we have found that explicit measures are often necessary to avoid starvation. Obstruction-free synchronization encourages a clean distinction between the obstruction-free mechanisms that ensure correctness (such as conflict detection and recovery) and additional mechanisms that ensure progress (such as adaptive backoff or queuing).

In our transactional memory implementation, progress is the responsibility of the contention manager. Each thread has its own contention manager instance, which it consults to decide whether to force a conflicting thread to abort. In addition, contention managers of different threads may consult one another to compare priorities and other attributes.

The correctness requirement for contention managers is simple and quite weak. Informally, any active transaction that asks sufficiently many times must eventually get permission to abort a conflicting transaction. More precisely,

${ }^{8} \mathrm{~A}$ further optional step can reduce space overhead by storing null to whichever of the object pointers in a locator becomes obsolete after its transaction either commits or aborts, thereby allowing the garbage collector to claim it. (Straightforward changes would be required in order to avoid dereferencing these null pointers.) 
every call to a contention manager method eventually returns (unless the invoking thread stops taking steps for some reason), and every transaction that repeatedly requests to abort another transaction is eventually granted permission to do so. This requirement is needed to preserve obstructionfreedom: A transaction $T$ that is forever denied permission to abort a conflicting transaction will never commit even if it runs by itself. ${ }^{9}$ If the conflicting transaction is also continually requesting permission to abort $T$, and incorrectly being denied this permission, the situation is akin to deadlock. Conversely, if $T$ is eventually allowed to abort any conflicting transaction, then $T$ will eventually commit if it runs by itself for long enough.

The correctness requirement for contention managers does not guarantee progress in the presence of conflicts. Whether a particular contention manager should provide such a guarantee-and under what assumptions and system models it should do so--is a policy decision that may depend on applications, environments, and other factors. The problem of avoiding livelock is thus delegated to the contention manager.

Rather than mandate a specific contention-management policy, we define a ContentionManager interface that every contention manager must implement. This interface specifies two kinds of methods, notification methods and feedback methods, which are invoked by our DSTM implementation.

Notification methods inform a contention manager of relevant events in the DSTM; they do not return any value. For example, the commitTransactionsucceeded() method is invoked whenever a transaction commits successfully, and the commitTransactionfailed() method is invoked whenever an attempt to commit a transaction fails. Some notification methods correspond to events internal to our DSTM implementation. For example, the openReadAttempt () method is called to notify a contention manager before any attempt to open in READ mode an object that is not already open; similarly, the openWriteAttempt () method is called before any attempt to open an object in WRITE mode.

Feedback methods are called by the DSTM to determine what action should be taken in various circumstances. One important feedback method is shouldabort (), which is invoked whenever the DSTM detects a conflicting transaction during an attempt to open an object. The shouldabort () method is passed the object being opened and the manager of the conflicting transaction, and it returns a boolean indicating whether to try to abort the conflicting transaction.

In addition to their explicit purposes, the contention manager's methods may implement other measures, such as backoff and queuing, to manage contention. We have done only preliminary work using these methods to implement some simple contention management strategies, and we expect the ContentionManager interface to evolve as we gain experience with what methods especially notification methodsare useful for implementing more sophisticated strategies.

\subsection{Examples}

As a baseline for the experimental results reported in Section 5, we implemented a trivial Aggressive contention manager that always and immediately grants permission to abort any conflicting transaction. We also implemented a

\footnotetext{
${ }^{9}$ Here and elsewhere "runs by itself" means that no concurrent transaction takes a step, not that no concurrent transaction exists.
}

simple Polite contention manager, that adaptively backs off a few times when it encounters a conflict. Specifically, when a transaction first invokes shouldabort () for an object, the method sleeps for a random duration before returning false, refusing permission to abort the other thread. Each subsequent call to shouldabort () for the same object doubles the expected sleep time, until a threshold is reached. Beyond that threshold, shouldabort() returns immediately and returns true, granting the caller permission to abort the conflicting transaction.

One can imagine many variations on this strategy, as well as different strategies based on queuing rather than backoff combined with spinning. Discovering which strategies work best remains an open area of research.

\section{RESULTS}

In this section, we briefly present the results of some simple performance experiments we conducted on a Sun Fire ${ }^{\mathrm{TM}}$ $15 \mathrm{~K}$ server with $721050 \mathrm{MHz}$ SPARC $(\mathrm{B}$ processors.

In each experiment, we implemented an integer set and measured how many operations completed on the integer set in 20 seconds, varying the number of participating threads between 1 and 576 (a multiprogramming level of 8 ). For each operation, we randomly choose a value between 0 and 255 and randomly choose whether to insert or delete the value. The restricted range ensures significant contention among concurrent threads, and thus exercises the contention managers. In each experiment, each thread executes operations repeatedly with no delay between them in order to examine how the implementations scale with increased contention.

The results of our experiments are presented in Figure 6. The graphs show results as throughput in operations per millisecond. Each point represents the average of at least ten runs of the relevant experiment. The upper graph shows the results for the various experiments, running from 1 to 576 threads. The lower graph presents a more detailed look at the experiments in which the number of threads does not exceed the number of processors (72). Of course, many more experiments should be conducted to test various implementation approaches at the transaction, contention manager, and STM levels. The simple experiments presented here are intended only to illustrate some broad principles.

We first implemented a simple linked list synchronized with a single lock (see "Simple Locking" in Figure 6). Due to its simplicity, this implementation yields a higher throughput than any other configuration in the single-threaded case (768 operations per millisecond). However, as the number of threads increases, the throughput of this implementation quickly falls off; in particular, when there are more threads than processors, this implementation performs very badly due to preemption while holding the lock. ${ }^{10}$

Next, we used DSTM to implement the simple transactional integer set shown in Figure 1. When composed

${ }^{10}$ There are specialized optimistic locking algorithms that exploit the simple semantics of linked lists to substantially improve performance. However, these algorithms involve unsynchronized reads of shared data, and thus require careful reasoning about concurrency to ensure correctness and avoid deadlock. Furthermore, these algorithms do not generalize straightforwardly to more complex data structures. Because our purpose here is to illustrate the implications of different implementation approaches, not to construct the best implementation of integer sets, we do not consider such algorithms in this paper. 

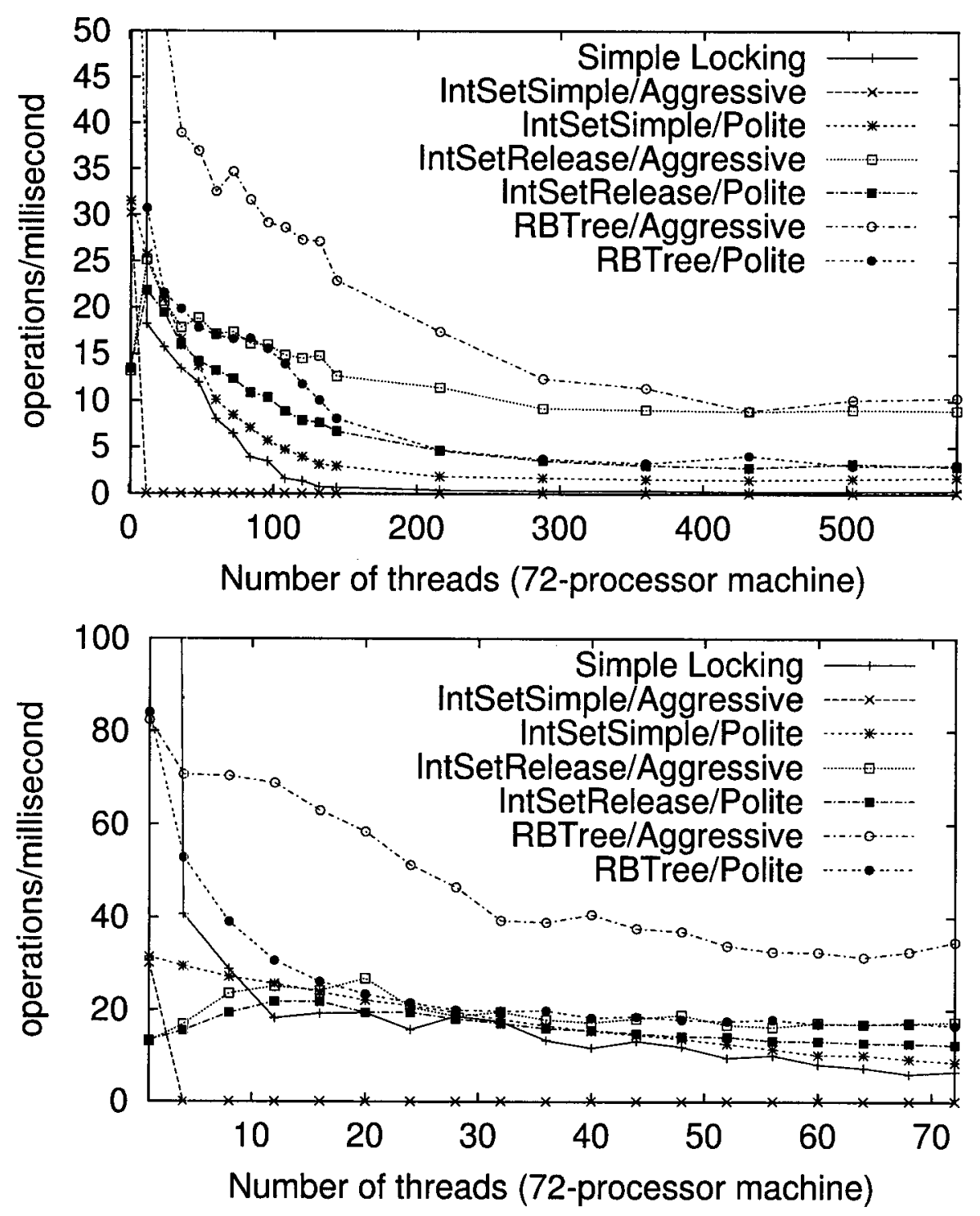

Figure 6: Experimental results on 72-processor Sun Fire 15K server. The bottom figure shows a detailed view of the top one for up to 72 threads.

with the trivial Aggressive contention manager (IntSetSimple/Aggressive in Figure 6), this implementation immediately livelocks as soon as there is more than one thread. However, when we compose the same implementation with the slightly more sophisticated P'olite contention manager, it performs much better. In fact, it outperforms the simple lock-based implementation when there are more than about 10 threads. These results demonstrate the necessity and effectiveness of contention management.

As discussed in Section 2, it is often preferable to avoid contention rather than merely manage it. We therefore also tested the linked list implementation with early release, as shown in Figure 2. This implementation greatly reduces contention because a transaction has many fewer objects open at any time. As seen in Figure 6, this implementation does not livelock even when used with the Aggressive contention manager (IntSetRelease/Aggressive in Figure 6), demonstrating that this programming technique is effective at reducing contention. Because this implementation gives rise to less contention, the effect of contention management is less pronounced. Interestingly, however, the early release implementation performs better with the Aggressive contention manager than with the Polite one, especially when the number of threads exceeds the number of processors. One possible explanation for this difference is that we have not tuned the Polite manager for the case in which there is no contention. Also, when there are more threads than processors, a transaction might conflict with another transaction whose thread is preempted, in which case, it may be best to abort that other transaction immediately. We have yet to conduct more detailed experiments to test these conjectures and fully understand the cause of this effect.

In the context of sequential algorithms, it is standard practice to design more complex algorithms that outperform 
simpler ones (for example, by implementing a balanced tree instead of a list). For non-blocking algorithms, however, implementing more complex data structures has been prohibitively difficult. Our work on DSTM makes a significant step towards overcoming this difficulty. To demonstrate, we have used DSTM to implement a non-blocking red-black tree using a straightforward translation from sequential code [6]. To reduce contention, our red-black tree implementation initially opens nodes in READ mode, upgrading to WRITE mode as needed. To keep it simple, we do not release any nodes until the transaction commits (or is aborted). We tested our red-black tree implementation with the Aggressive and Polite contention managers (RBTree/Aggressive and RBTree/Polite in Figure 6).

As can be seen from Figure 6, our red-black tree significantly outperforms the other non-blocking implementations at low levels of contention (fewer than ten threads). We expected this improvement because the red-black tree's time complexity is logarithmic in the size of the set, in contrast to the linear time complexity of the list. This effect would be even more pronounced if we chose values to insert from a larger range, resulting in larger sets in the steady state.

Even with this limited value range, the red-black tree using the Aggressive contention manager significantly outperforms all other configurations at most levels of contention, although there is a marked degradation in its performance as the number of threads increases. With the Polite contention manager, the red-black tree does not perform quite as well, but it remains competitive with all of the other configurations shown while we have at most one thread per processor, and is significantly better than most of them.

These observations suggest several lessons. First, unsurprisingly, sophisticated data structures, such as red-black trees, can significantly outperform simpler data structures, such as linked lists. Our DSTM makes it relatively straightforward to transform sequential algorithms into non-blocking ones, and thus, allows us to leverage decades of work on efficient sequential data structures in our development of non-blocking data structures. Second, the difference in performance between configurations with different contention managers reinforces the importance of contention management for designing efficient non-blocking data structures. Finally, the performance degradation of the red-black tree with both contention managers suggests that there is room for considerable improvement with more sophisticated contention managers that impose very low overhead when contention is not a problem, but manage contention better when it is.

One shortcoming of our current DSTM implementation with respect to the range of possible contention managers is that there is no way for one transaction to detect that another transaction has opened an object in READ mode. By opening that object in WRITE mode, the first transaction will cause the other transaction to abort. Clearly there is a tradeoff between the amount of synchronization needed to open an object for reading in a "visible" way in order to enable competing transactions to "be polite" and the benefit derived from doing so. We are currently working on some ideas in this direction.

\section{CONCLUDING REMARKS}

We have proposed a new form of dynamic software transactional memory (DSTM), which supports relatively straightforward programming of a wide variety of dynamicsized data structures. For example, we have used it to implement a non-blocking red-black tree, by far the most sophisticated non-blocking data structure achieved to date. We have implemented an obstruction-free prototype of our DTSM in the Java programming language. Obstruction-freedom is a new non-blocking progress condition we proposed recently; it is weaker than previous such conditions, and as a result, admits substantially simpler implementations.

An attractive feature of our implementation is the ability for a transaction to detect that it will cause another to abort before it does so, and therefore decide whether to proceed or to give the other transaction a chance to complete first. Such policy decisions are made by modular contention managers that can be "plugged in" without affecting the transaction code or its correctness. Preliminary performance experiments show that nontrivial contention management schemes are necessary in order to avoid livelock, and that even simple schemes can be effective.

We have only begun to explore the range of possible contention manager designs. We believe that designing, testing, and reasoning about modular contention managers will be a rich source of research problems. It is interesting to note that it is possible to design contention managers that make provable progress guarantees in the presence of certain weak but reasonable assumptions about the underlying system (and the transaction code). Whether such managers are practical is a matter for future research.

Another interesting and novel feature of our DSTM is the ability to "release" objects from a transaction before it commits. This feature puts significantly more burden on the transaction programmer in reasoning about correctness, but can also provide considerable performance improvements when used with care.

Some interesting issues also remain regarding interface and semantics. In many cases, there are tradeoffs between efficiency of implementation and usability and simplicity of interface; we have yet to explore these tradeoffs in detail.

Acknowledgments: Thanks to Ron Larson for getting us access to the Sun Fire $15 \mathrm{~K}$ computer, to Doug Lea for his experimental java.util.concurrent package, to Steve Green for his help with the experiments, and to Steve Heller for useful discussions. Thanks also to Guy Steele and Jan-Willem Maessen for useful feedback, especially about the DSTM interface. (Jan also suggested nulling out the extra pointer of locators whose transactions are aborted or committed to allow garbage collection of the obsolete version.) We are also grateful to Yossi Lev for feedback and useful suggestions for future improvements, and to Mike Kistler for his comments on a recent draft of this paper.

\section{REFERENCES}

[1] Java Specification Request for Concurrent Utilities (JSR166). http://jcp.org.

[2] Sun Microsystems Laboratories Scalable Synchronization Research Group publications page. http://research.sun.com/scalable/pubs.

[3] Y. Afek, D. Dauber, and D. Touitou. Wait-free made fast. In Proceedings of the 27th Annual ACM Symposium on Theory of Computing, pages 538-547, 1995. 
[4] G. Barnes. A method for implementing lock-free shared data structures. In Proceedings of the Fifth Annual ACM Symposium on Parallel Algorithms and. Architectures, pages 261-270, 1993.

[5] R. Bayer and M. Schkolnick. Concurrency of operations on B-trees. Acta Informatica, 9:1-21, 1977.

[6] T. H. Cormen, C. E. Leiserson, and R. L. Rivest. Introduction to Algorithms. McGraw-Hill, 1990.

[7] M. Herlihy, V. Luchangco, and M. Moir. Obstruction-free synchronization: Double-ended queues as an example. In Proceedings of the 23rd International Conference on Distributed Computing Systems, 2003.

[8] M. Herlihy and J. Moss. Transactional memory: Architectural support for lock-free data structures. In Proceedings of the 20th International Symposium in Computer Architecture, pages 289-300, 1993.

[9] M. Herlihy and J. Wing. Linearizability: A correctness condition for concurrent objects. ACM Transactions on Programming Languages and Systems, 12(3):463-492, 1990.

[10] A. Israeli and L. Rappoport. Disjoint-access-parallel implementations of strong shared memory primitives. In Proceedings of the 13th Annual ACM Symposium on Principles of Distributed Computing, pages 151-160, 1994.

[11] V. Luchangco, M. Moir, and N. Shavit. Nonblocking $k$-compare-single-swap. In Proceedings of the 15th Annual ACM Sympoium on Parallel Architecures and Algorithms, 2003.

[12] M. Michael and M. Scott. Nonblocking algorithms and preemption-safe locking on multiprogrammed shared memory multiprocessors. Journal of Parallel and Distributed Computing, 51(1):1-26, 1998.

[13] M. Moir. Transparent support for wait-free transactions. In Proceedings of the 11th International Workshop on Distributed Algorithms, pages 305-319, 1997.

[14] N. Shavit and D. Touitou. Software transactional memory. Distributed Computing, Special Issue(10):99-116, 1997.

[15] J. Turek, D. Shasha, and S. Prakash. Locking without blocking: making lock basea concurrent data structure algorithms nonblocking. In Proceedings of the eleventh ACM SIGACT-SIGMOD-SIGART symposium on Principles of database systems, pages 212-222, 1992. 